\title{
SEQUELAS BUCAIS DA RADIOTERAPIA DE CABEÇA E PESCOÇO
}

\section{Oral sequelae of head and neck radiotherapy}

\author{
Daniel Antunes Freitas ${ }^{(1)}$, Antonio Diaz Caballero(2), Mayane Moura Pereira(3), \\ Stephany Ketllin Mendes Oliveira ${ }^{(4)}$, Gracielle Pinho E Silva ${ }^{(5)}$, Clara Inés Vergara Hernández ${ }^{(6)}$
}

\section{RESUMO}

Tema: cirurgia, radioterapia e quimioterapia são as modalidades terapêuticas usadas no tratamento de câncer bucal. Podem ser usadas isoladas ou conjuntamente. Radiação ionizante causa lesões nos tecidos normais localizados no campo de radiação. Isto se torna particularmente evidente nas regiões de cabeça, uma área complexa composta de várias estruturas diferentes que respondem diferentemente à radiação. As seqüelas orais resultantes podem causar problemas substanciais durante e depois da terapia de radiação e são os maiores fatores de determinação na qualidade de vida dos pacientes. Dentre as complicações da radioterapia estão a xerostomia, osteorradionecrose, mucosite e candidose. Objetivo: apresentar aos profissionais de saúde uma reflexão sobre as questões pertinentes às sequelas bucais da radioterapia de cabeça e pescoço. Conclusão: o acompanhamento odontológico sistemático pode minimizar os efeitos da radiação sobre os tecidos da cavidade bucal.

DESCRITORES: Radioterapia; Câncer; Saúde Bucal

\section{INTRODUÇÃO}

O câncer de cabeça e pescoço, que em nível mundial representa cerca de $10 \%$ dos tumores malignos, envolve vários sítios, sendo que uma média de $40 \%$ dos casos ocorrem na cavidade oral, $25 \%$ na laringe, $15 \%$ na faringe, $7 \%$ nas glândulas salivares e $13 \%$ nos demais locais. Anualmente

(1) Odontólogo. Mestre em Odontologia; Editor Científico da Revista AFROUNIMONTES; Professor Titular dos Cursos de Fonoaudiologia e Odontologia das Faculdades Unidas do Norte de Minas - FUNORTE, Montes Claros, MG, Brasil.

(2) Odontólogo. Mestre em Educação; Consultor Científico Internacional da Revista AFROUNIMONTES; Professor Titular do Curso de Odontologia da Universidade de Cartagena - Cartagena, Colômbia.

(3) Acadêmica e jovem-pesquisadora do Curso de Odontologia das Faculdades Unidas do Norte de Minas - FUNORTE, Montes Claros, MG, Brasil.

(4) Acadêmica e jovem-pesquisadora do Curso de Odontologia das Faculdades Unidas do Norte de Minas - FUNORTE, Montes Claros, MG, Brasil.

(5) Acadêmica e jovem-pesquisadora do Curso de Odontologia das Faculdades Unidas do Norte de Minas - FUNORTE, Montes Claros, MG, Brasil.

(6) Odontóloga. Especialista em Patologia e Cirurgia Oral. Vice-Diretora e Professora Titular do Curso de Odontologia da Universidade de Cartagena - Cartagena, Colômbia.

Conflito de interesses: inexistente ocorrem mais de oito milhões de casos novos de câncer no mundo, dos quais mais de 200.000 originam-se na boca. Segundo estimativas do INSTITUTO NACIONAL DO CÂNCER (INCA-BRASIL), em 2010 serão 14.120 novos casos, sendo 10.330 homens e 3.790 mulheres em todo o Brasil, sendo que em 2008 morreram 6.214 pessoas com câncer bucal, sendo 4.898 homens e 1.316 mulheres $^{1-3}$.

A radioterapia de cabeça e pescoço e a cirurgia são os tratamentos mais usados no combate a este tipo de câncer. Os pacientes que são submetidos a tratamento radioterápico em região de cabeça e pescoço, freqüentemente, desenvolvem alterações e seqüelas de interesse da estomatologia.

As condições desfavoráveis mais comuns são xerostomia, osterradionecrose, mucosite e candidose. A xerostomia é a sensação de boca seca, que pode ser causada por uma diminuição ou não da função das glândulas salivares, com alteração quer na quantidade,quer na qualidade da saliva. A osteorradionecrose é a complicação decorrente da radiação ionizante que atinge maxila e/ ou mandíbula, é uma grande preocupação devido seu comportamento agressivo. A mucosite é a inflamação da mucosa oral, acomete primariamente os pacientes e provoca picos de dor. A candidose é o surgimento patológico de lesões a partir do desenvolvimento de microorganismos tipo Cândida ${ }^{1,2,4}$. 
O profissional de Odontologia, bem como médicos oncologistas e fonoaudiólogos da área hospitalar, devem estar cientes destes distúrbios bucais decorrentes da radioterapia para que possam ajudar os pacientes a obter melhor qualidade de vida durante seu tratamento. É de fundamental importância, que os profissionais envolvidos neste processo, estejam revestidos do conhecimento científico adequado e do oportuno senso de interdisciplinaridade, qualidades obrigatórias a uma atenção eficiente. Dessa forma, o objetivo deste trabalho é apresentar aos profissionais de saúde uma reflexão sobre as questões pertinentes às sequelas bucais da radioterapia de cabeça e pescoço.

\section{MÉTODO}

Para o desenvolvimento deste estudo sobre saúde bucal e radioterapia de cabeça e pescoço, foram realizadas buscas de literatura científica nas seguintes bases de dados / portais de pesquisa: Pubmed/Medline, Scielo, LILACS e BIREME. Os descritores e expressões utilizados durante as buscas nas bases de dados foram: oral health, head and neck radiotherapy, ionizing radiation, oral candidosis, radiotherapy, mucositis, xerostomia, osteoradionecrosis, salivary glands, hyposalivation. Foram utilizados artigos publicados nos últimos 10 anos, correspondem ao período entre o ano 2000 e o ano 2010, preferencialmente em inglês, que apresentassem relevância relativa ao tema pesquisado.

\section{REVISÃO DE LITERATURA}

\section{Radioterapia de cabeça e pescoço}

A radioterapia é feita de forma ionizante, o meio iônico é ionizado tornando-se eletricamente instável. Essa radiação vai agir sobre o DNA nuclear levando a perda de sua capacidade reprodutiva ou a morte. O DNA duplica durante a mitose, nisto as células com grande capacidade de duplicação são mais radiossensíveis. Por estarem em constante atividade mitótica às células neoplasias são mais susceptíveis às radiações. A dose necessária de radiação varia conforme a malignidade e localização da neoplasia ${ }^{2,4-6}$.

A maioria dos pacientes são tratados com a dose total curativa entre 50 e 70 Gy, sendo fracionada num período de 5- 7 semanas, 5 dias por semana, uma vez ao dia, sendo $2 \mathrm{~Gy}$ por fração. A dose total da radioterapia para linfomas malignos é normalmente menor. A radiação fracionada permite um efeito preservador, dando uma resposta tardia, no entanto a resposta precoce responde melhor ao tumor, permite a repopulação de tecido entre as frações, reduzindo efeitos precoces, permite também a reoxigenação dos tumores hipóxicos radio-resistentes entre frações, permitindo uma maior porcentagem de células radiosensíveis oxigenadas. $O$ fator de limitação da dose mais importante é a tolerância dos tecidos normais adjacentes. Tecidos com rápido índice de retorno mostram efeitos precoces à radioterapia, enquanto nos tecidos de menor índice de retorno acontecem efeitos tardios á radioterapia. A fracionação acelerada é baseada na observação que a lesão por radiação causa acelerada proliferação dos tecidos do tumor, diminuindo o tempo de tratamento se resolveria esse problema. As frações são dadas tipicamente duas vezes por dia. A hiperfracionação tem sido uma alternativa da radioterapia para minimizar os efeitos colaterais, sendo que ela faz uso da diferença entre as capacidades de reparação ante ao tumor pelo fracionamento da dose, sendo que o tempo de tratamento convencional deve ser mantido onde 02 frações por dia são dadas. Com este tipo de fracionação, o total que o tumor absorve da dose pode ser aumentado enquanto não houver toxidade. As combinações de hiperfracionação e programas acelerados têm se mostrado bem sucedidos para tumores que se dividem rapidamente, as desvantagens são os efeitos colaterais, especialmente a mucosite ${ }^{5-9}$.

A terapia em 3D é uma recente técnica radioterápica, onde se podem distribuir espacialmente altas doses de radiação para um volume alvo, reduzindo o dano em tecidos normais, todavia necessita de mais pesquisas sobre o método ${ }^{2,5,9}$.

\section{Xerostomia}

A radioterapia aplicada na região da cabeça e do pescoço apresenta entre seus efeitos indesejáveis a xerostomia ${ }^{3,10-12}$.

A saliva desempenha um papel fundamental na manutenção da homeostase da cavidade bucal. É um dos mais complexos, versáteis e importantes fluídos do corpo, que supre um largo espectro de necessidades fisiológicas. As suas propriedades são essenciais para a proteção da cavidade bucal e do epitélio gastrointestinal. Noventa por cento da saliva é produzida pelas glândulas salivares major, sendo a restante produzida pelas glândulas salivares da mucosa da boca e faringe, seu volume diário produzido situa-se entre 0,5 a 1,5 litros de saliva e seu pH oscila entre os 6,5-7,4, 4,11,13.

As principais funções da saliva são: a) relacionadas com a fase líquida - limpeza da cavidade oral, limpeza dos restos alimentares e bactérias, solubilização de substâncias alimentares contribuindo para a percepção do paladar, lubrificação da mucosa oral, facilitação da mastigação, da deglutição e da 
fonação; b) relacionadas com os solutos-proteção dos dentes, neutralização dos ácidos, participação na formação do esmalte, proteção da mucosa oral e sua cobertura, defesa contra micro-organismos e ação digestiva. Foi ainda sugerido que os sistemas tampão da saliva auxiliariam a controlar os efeitos do refluxo do conteúdo gástrico para o esôfago ${ }^{11,14}$.

A diminuição efetiva da quantidade do fluxo salivar é denominada hipossalivação, enquanto que a xerostomia é a sensação subjetiva de boca seca, consequente ou não da diminuição da função das glândulas salivares, com alterações quer na quantidade, quer na qualidade da saliva, é um sintoma frequente em doentes em cuidados paliativos. Pacientes sem alterações no fluxo salivar podem se queixar de secura na boca. Os pacientes com xerostomia queixam-se de desconforto bucal, perda do paladar, dificuldades na fala e deglutição $0^{3,13}$.

A extensão da lesão induzida pela radioterapia depende do volume de glândulas irradiado, em especial das parótidas, da dose total e da técnica utilizada. Habitualmente assiste-se a uma fase aguda de xerostomia causada pela radioterapia que surge logo à primeira semana, mas também pode haver um efeito mais tardio e permanente de compromisso da função. Ou seja, após alguma recuperação da secreção salivar esta pode regredir mais tardiamente e de modo irreversível ${ }^{2,12,14}$.

A saliva sofre, também, alterações qualitativas decorrentes da radioterapia com diminuição da atividade das amilases, capacidade tampão e pH, com conseqüente acidificação. Ocorrem também alterações dos diversos eletrólitos como cálcio, potássio, sódio e fosfato. Desta forma, os indivíduos que foram irradiados são mais susceptíveis à doença periodontal, cáries rampantes e infecções bucais fúngicas e bacterianas. As alterações produzidas nas glândulas salivares incluem degeneração acinosa e adiposa, além de fibrose com acentuada redução do fluxo salivar e aumento da viscosidade da saliva. A secreção salivar diminui drasticamente quando as glândulas salivares estão incluídas no campo de radiação. Sua redução está relacionada com a dose e à duração terapêutica da radiação induzida e reflete mudanças inflamatórias e degenerativas nos ácinos e células dos ductos. $\mathrm{Na}$ glândula irradiada, o arranjo celular dos ductos é substituído por remanescentes do tecido conjuntivo frouxo e fibroso moderadamente infiltrado com linfócitos e plasma celular ${ }^{3,10,11-14}$.

O tratamento da xerostomia é essencialmente paliativo. É descrita uma relação direta entre a dose de irradiação e a extensão das modificações glandulares, pode ser feito por meio do uso de estimulantes mecânicos/gustatórios, substitutos da saliva ou agentes sistêmicos. Métodos alternativos, como a acupuntura, também já foram citados como forma de tratamento da xerostomia. Estimulantes e substitutos da saliva geralmente atenuam apenas a xerostomia, sem alterar o fluxo salivar. Já os agentes sistêmicos além de atenuar a xerostomia, diminuem também os problemas bucais associados com a hipofunção das glândulas salivares, por meio da elevação do fluxo salivar. Desta forma, o tratamento de escolha da xerostomia associada à radioterapia deve ser por meio do uso de agentes sistêmicos, sendo que a pilocarpina é o mais estudado. Estudos mostram que os agentes sistêmicos, como a pilocarpina, são mais eficazes quando usados durante a radioterapia. Recentemente, isto foi demonstrado também para o betanecol, sendo que o medicamento usado concomitantemente à radioterapia é capaz de aumentar o fluxo salivar em repouso logo após o término do tratamento radioterápico, além de diminuir a queixa subjetiva de boca seca $^{2,11,13,14}$.

\section{Osteorradionecrose}

Após o tratamento de radiação o tecido sofre várias alterações se tornando hipóxico, hipovascular e hipocelular prejudicando a reconstituição do osso e favorecendo a ocorrência da osteorradionecrose. Alterações ósseas podem ser observadas em casos de procedimentos durante o primeiro ano e segundo e quinto ano após radioterapia, mas há relatos de ocorrência de 3-7 meses e 38-45 anos após radioterapia ${ }^{2,10,12,15-17 . ~}$

A osteorradionecrose pode apresentar como características clínicas: ulceração da mucosa com exposição óssea e presença de dor, sendo a mandíbula mais acometida que a maxila. $\mathrm{O}$ diagnóstico se dá por meio da avaliação clínica e radiográfica devido a presença de áreas de reabsorção e neoformação óssea ${ }^{2-4,12,15,18}$.

A exodontia em pacientes que sofreram tratamento por radioterapia constitui uma preocupação, uma vez que é alto o risco de necrose óssea. Para dentes com indicação de exodontia temos como escolha a utilização de elásticos ortodônticos promovendo avulsão dos mesmos por um processo menos traumático. Entretanto, dentes fortemente aderidos ao osso e com múltiplas raízes só são removidos empregando-se a técnica convencional sendo necessária a oxigenação hiperbárica antes do procedimento e manutenção após o término ${ }^{3,12,16,19}$.

A avaliação odontológica pré-radioterápica é fundamental, pois permite o levantamento das necessidades e tratamento odontológico do paciente antes do início da radioterapia. Nessa avaliação, o cirurgião dentista deve incluir procedimentos como raspagem de tártaro, eliminação de 
cáries, orientação sobre higiene oral e exodontias necessárias permitindo a manutenção da saúde bucal do paciente e a prevenção da osteorradionecrose $^{2,15-19}$.

\section{Mucosite}

Mucosite Oral é a forma mais comum de complicação oral decorrente de terapia antineoplásica não-cirúrgica; é caracterizada pelo aparecimento precoce de lesões orais, dolorosas e debilitantes. Pode aparecer induzida por drogas citotóxicas e principalmente pela radiação de cabeça e pescoço. É encontrada em $40 \%$ dos pacientes que recebem a quimioterapia e em $100 \%$ dos pacientes que recebem radioterapia de cabeça e pescoço $0^{2-4,10,20-22}$.

As superfícies epiteliais da mucosa oral estão em freqüente renovação celular, em que há perdas freqüentes de células da superfície epitelial devido a traumas mecânicos, sendo compensada pela contínua proliferação das células da camada basal. O desequilíbrio entre a proliferação e a perda celular produz redução das células epiteliais, resultando em um epitélio com menor espessura, o que se manifesta como mucosite na mucosa oral. Essa proliferação ocorre três vezes mais em crianças do que em adultos ${ }^{20,22}$.

A Mucosite Oral pode ser classificada como:

0 - ausente (mucosa e gengiva estão úmidos e róseos);

1 - descoloração, aspecto esbranquiçado, possibilitando dieta normal;

2 - eritema, possibilitando dieta normal;

3 - pseudomembrana, requerendo dieta líquida;

4 - ulceração profunda, que impossibilitava a alimentação oral ${ }^{2,12,20,23}$.

A mucosa oral passa por uma série de mudanças que estão relacionadas com a dose e duração do tratamento. A primeira reação da mucosa bucal à radiação é edema e eritema devido à dilatação vascular da mucosa está intacta apenas com uma ardência local. Posteriormente, a mucosa tornase desnuda, ulcerada e recoberta com exsudato fibrinoso, além da perda de espessura. Dor, queimação e desconforto são comumente presentes em pacientes em repouso e são intensificados pelo contato com alimentos duros e muito temperados. O envolvimento da faringe produz dificuldade para engolir e falar. $\mathrm{Na}$ língua, podem ocorrer atrofia nas papilas, inflamação, fissuras e erosões e, em casos mais severos, áreas de desnudação. Em sua evolução, pode-se observar a odinofagia, disfagia e infecção por microorganismos oportunistas. Pode também interferir na manutenção de uma nutrição adequada pelo paciente, conduzindo a perda de peso, anorexia, caquexia e desidratação, restringindo, ainda mais, a sua dieta a líquidos ou, até mesmo, levando à indicação de sonda nasogástrica, o que compromete o seu estado geral de saúde. As percepções de sabores amargos e ácidos são mais acometidas que os sabores doces e salgados. Os pacientes atribuem depressão e distúrbios do sono a mucosite. Faz-se necessário o controle dos sintomas, pois, um bom estado de saúde geral e uma boa nutrição interferem na saúde emocional do paciente, e em seu sistema imune, tornando o paciente mais resistente a infecções e a própria neoplasia ${ }^{2,4,20,21}$.

Os pacientes devem ter cuidados orais especiais, tais como, limpeza dos dentes com escova macia, creme dental de preferência com flúor, cuidados com próteses (limpeza e ajustes), avaliação da presença de cáries, uso de fio dental, nutrição adequada, evitando alimentos ácidos, muito condimentados e açúcar, e a manutenção de uma hidratação adequada. O bochecho de bicarbonato de sódio cria um ambiente alcalino, impedindo a proliferação de candidíase; no entanto, pode ser desagradável no paladar. A solução salina 0,9\% não é irritante e não modifica o pH da saliva, além de ser econômica é recomendada. O peróxido de hidrogênio, apesar de controvérsias, ainda é utilizado, porém causa irritação, dano ao tecido de granulação, interrompe a flora normal da cavidade oral e pode causar náuseas devido ao paladar. Suspensões com magnésio e com hidróxido de alumínio são soluções que protegem a mucosa, formando uma camada com efeito analgésico, minimizando a acidez, porém ressecam a mucosa oral, necessitando, pois, de mais pesquisas. Bochechos com nistatina, para a prevenção contra fungos, também são recomendados, antes de se iniciar o tratamento, três vezes ao dia por sete dias e fluoreto de sódio (gel) $0,05 \%$, diariamente. O gluconato de clorexidina $0,12 \%$ também é um recomendado antimicrobiano. O palifermin é um fator de crescimento de queratinócitos recombinante, aprovado na Europa e recentemente nos Estados Unidos, para a redução e incidência da mucosite oral em malignidades hematológicas. Uma variedade de técnicas em terapia alternativa pode ser utilizada: técnicas de distração, programas de treinamento cognitivo, musicoterapia, técnicas de relaxamento e massagem, técnicas de imagem e outras. A acupuntura tem sido considerada, mas necessita ainda de mais estudos $2,3,10,20,22,23$.

Bochechos periódicos com anestésicos tópicos com xilocaína e benzidamina tem sido propostos. Administração de antibióticos tem mostrado ser de algum uso na redução da severidade da mucosite por radiação. Doxepin tópico tem mostrado ser efetivo em severa mucosite nos pacientes recebendo radiação. A diminuição da resposta 
inflamatória decorrente da radioterapia pode ser resolvida com uso de agentes antiinflamatórios, como a pentoxifilina e a indometacina. A capsaicina, um potente inibidor de dor neuropática, tem demonstrado em vários estudos a redução da dor oral em pacientes que experimentam mucosite oral durante o curso de radioterapia. A interleucina-11 (IL-11) inibe fatores pró-inflamatórios como a interleucina- 12 (IL-12) ${ }^{3,10,21,23 .}$

O laser de baixa intensidade vem sendo utilizado como forma de tratamento e cicatrização da mucosite oral e tem obtido respostas positivas do ponto de vista clínico e funcional. As células conduzem à liberação de fatores de crescimento por macrófagos, proliferação de queratinócitos, aumento da população e de granulação de mastócitos e angiogênese $e^{2,20-23}$.

\section{Candidose}

A candidose oral tem como principal agente etiológico a Candida albicans. Sua manifestação clínica se caracteriza pela presença de placas brancas removíveis a raspagem, podendo apresentar-se na forma pseudomembranosa ou eritematosa ${ }^{2,3,10,24-26}$.

Apesar de ser um componente da flora bucal normal, alguns fatores podem favorecer o crescimento desse patógeno oportunista, provocando infecção na mucosa oral, vaginal ou doença sistêmica. O paciente irradiado apresenta uma queda no fluxo salivar o que pode justificar o aumenta na ocorrência da candidose, geralmente também asso-

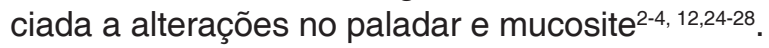

O diagnóstico se dá por meio do exame clínico e seu tratamento consiste no emprego de antifúngicos tópicos ou sistêmicos sendo estes a nistatina e o fluconazol ${ }^{3,10,25-27 . ~}$

A manutenção da saúde bucal e o acompanhamento odontológico regular podem reduzir e prevenir de forma considerável a ocorrência da candidose em pacientes que passam por radioterapia em área de cabeça e pescoço-4, 10, 12,24-28.

\section{CONCLUSÃO}

A radioterapia pode provocar efeitos indesejáveis sobre o organismo humano, e estes efeitos ocorrem com freqüência entre os pacientes submetidos à radioterapia de cabeça e pescoço. Médicos oncologistas e fonoaudiólogos da área hospitalar devem apoiar na detecção e abordagem destas sequelas. Afecções bucais são esperadas para estes pacientes, cabendo ao profissional de Odontologia orientar e intervir para proporcionar mais qualidade de vida a estas pessoas. Fonoaudiólogos, médicos e odontólogos devem atuar com harmonia e extrema atenção a estas reações do tratamento oncológico, para que possam oferecer aos pacientes radioterapizados uma melhor qualidade de vida.

\begin{abstract}
Background: surgery, radiotherapy and chemotherapy are therapeutic modalities used in the treatment of oral cancer. They can be used separately or in combination. Ionizing irradiation causes damage to normal tissues located at the radiation field. This becomes particularly evident in the head and neck region, a complex area composed of several dissimilar structures that respond differently to radiation. The resulting oral sequelae may cause substantial problems during and after radiation therapy and are major factors in determining the patient's quality of life. Xerostomia, osteoradionecrosis, mucositis and candidosis are some of radiotherapy's complications. Purpose: introduce health professionals to reflect on issues relevant to oral sequelae of head and neck radiotherapy. Conclusion: regular dental follow-up may reduce the effects of radiation in the tissues of the oral cavity.
\end{abstract}

KEYWORDS: Radiotherapy; Cancer; Oral Health

\section{REFERÊNCIAS}

1. Instituto Nacional de Câncer. Estimativas da incidência e mortalidade por câncer. Rio de Janeiro: INCA; 2010. [acesso em: 21 de setembro de 2010]. Disponível em URL: http://www.inca.gov.br/ estimativa/2010/.
2. Jham BC, Freire ARS. Oral complications of radiotherapy in the head and neck. Rev Bras Otorrinolaringol 2006;72(5):704-8.

3. Vissink A, Jansma J, Spijkervet FKL, Burlage FR, Coppes RP. Oral Sequelae of head and neck radiotherapy. Crit Rev Oral Biol Med 2003;14(3):199-212. 
4. Cardoso MFA, Novikoff F, Tresso A, Segreto RA, Cervantes $O$. Prevenção e controle das sequelas bucais em pacientes irradiados por tumores de cabeça e pescoço. Radiol Bras 2005;38(2):107-15. 5. Bhide SA, Nutting CM. Recent advances in radiotherapy. BMC Méd 2010;28(8):25.

6. Tao Y, Daly-Schveitzer N, Lusinchi A, Bourhis J. Advances in radiotherapy of head and neck cancers. Curr Opin Oncol. 2010;22(3):194-9.

7. Castadot $P$, Lee JA, Geets X, Grégoire V. Adaptive radiotherapy of head and neck cancer. Semin Radiat Oncol. 2010;20(2):84-93.

8. De Bree R, Leemans CR. Recent advances in surgery for head and neck cancer. Curr Opin Oncol. 2010;22(3):186-93.

9. Krstevska V. Radiotherapy and chemotherapy in locally advanced head and neck squamous cell carcinoma. J BUON. 2009;14(3):361-73.

10. Vissink A, Jansma J, Spijkervet FKL, Burlage FR, Coppes RP. Prevention and treatment of the consequences of head and neck radiotherapy. Crit Rev Oral Biol Med 2003;14(3):213-25.

11. Taweechaisupapong S, Pesee M, Aromdee C, Laopaiboon M, Wkhunkitti W. Efficacy of pilocarpine lozenge for post-radiation xerostomia in patients with head and neck cancer. Aust Dent Jo 2006;51(4):333-7.

12. Ord RA, Blanchaert RH JR. Current management of oral cancer. A multidisciplinary approach. J Am Dent Assoc 2001;132:19S-23S.

13. Shiboski $\mathrm{CH}$, Hodgson TA, Ship JA, Schiødt M. Management of salivary hypofunction during and after radiotherapy. Oral Surg Oral Med Oral Pathol Oral Radiol Endod 2007;103(suppl 1):S66 e1-S66.

14. Haveman C, Huber M. Xerostomia management in the head and neck radiation patient. Tex Dent J. 2010;127(5):487-504.

15. Jereczek-Fossa BA, Orecchia AR. Radiotherapy induced mandibular bone complications. Cancer Treat Rev. 2002;28(1):65-74.

16. Schwartz $H$, Kagan R. Osteoradionecrosis of the mandible: scientific basis for clinical staging. Am J Clin Oncol 2002;25(2):168-71.

http://dx.doi.org/10.1590/S1516-18462011005000071

RECEBIDO EM: 27/10/2010

ACEITO EM: 09/03/2011

Endereço para correspondência:

Daniel Antunes Freitas

Faculdades Unidas do Norte de Minas - FUNORTE

Avenida Osmane Brandao, s/n - Bairro JK

Montes Claros - MG

CEP: 39400-000

E-mail: danielmestradounincor@yahoo.com.br
17. Berger A, Bensadoun RJ. [Normal tissue tolerance to external beam radiation therapy: the mandible] Cancer Radiother. 2010;14(4-5):295-300.

18. Peterson DE, Doerr W, Hovan A, Pinto A, Saunders D, Elting LS, Spijkervet FK, Brennan MT. Osteoradionecrosis in cancer patients: the evidence base for treatment-dependent frequency, current management strategies, and future studies. Support Care Cancer. 2010;18(8):1089-98.

19. Oh HK, Chambers MS, Martin JW, Lim HJ, Park $\mathrm{HJ}$. Osteoradionecrosis of the mandible: treatment outcomes and factors influencing the progress of osteoradionecrosis. J Oral Maxillofac Surg. 2009;67(7):1378-86.

20. Ps SK, Balan A, Sankar A, Bose T. Radiation induced oral mucositis. Indian $\mathrm{J}$ Palliat Care. 2009;15(2):95-102.

21. Raber-Durlacher JE, Elad S, Barasch A. Oral mucositis. Oral Oncol. 2010;46(6):452-6.

22. Elting LS, Keefe DM, Sonis ST et al; BURDEN OF ILLNESS HEAD AND NECK WRITING COMMITTEE. Patient-reported measurements of oralmucositisinheadandneckcancerpatientstreated with radiotherapy with or without chemotherapy: demonstration of increased frequency, severity, resistance to palliation, and impact on quality of life. Cancer. 2008;113(10):2704-13.

23. Lalla RV, Sonis ST, Peterson DE. Management of oral mucositis in patients who have cancer.Dent Clin North Am. 2008;52(1):61-77.

24. Barbeau J, Seguin J, Goulet JP et al. Reassessing the presence of Candida albicans in denture- related stomatitis. Oral Surg oral Med Oral Pathol Oral Radiol Endod 2003;(1):51-9.

25. Zhao $\mathrm{X}$, Oh SH, Yeater KN, Hoyer LL. Analysis of the Candida albicans Als2p and Als $4 p$ adhesins suggests the potential for compensatory function within the Als family Microbiology 2005; 151:1619-30.

26. Motta-Silva AC, Aleva NA, Chavasco JK, Armond MC, França JP, Pereira LJ. Erythematous oral candidiasis in patients with controlled type II diabetes mellitus and complete dentures. Mycopathologia 2010;169(3):215-23.

27. Aleva NA, Birman EG, Afonso W JR, Chavasco JK, Paula CR, Ribeiro A, Pereira LJ. Erythematous candidosis in patients with complete dentures and HIV+/AIDS. Mycoses 2007;50(5):407-11.

28. Leung WK, Dassanayake RS, Yau JY, Jin LJ, Yam WC, Samaranayake LP. Oral colonization, phenotypic, and genotypic profiles of Candida species in irradiated, dentate, xerostomic nasopharyngeal carcinoma survivors. J Clin Microbiol 2000;38(6):2219-26. 